\title{
Identification of gas pipelines hydraulic efficiency coefficients
}

\author{
Andrey A. Belevitin ${ }^{1, *}$, and Victoria G. Ryzhkova ${ }^{1}$ \\ ${ }^{1}$ NIIgazeconomika LTD, 20-8, Staraya Basmannaya str., Moscow, Russia, 105066
}

\begin{abstract}
This study investigates the identification of non-measureable parameters of the gas transmission system (gas pipelines hydraulic efficiency coefficients). The problem statement and solution procedure are presented. The original problem is divided into two interrelated components: the nonlinear optimization problem and the temperature calculation. The nonlinear optimization problem is solved using the Successive Linear Programming (SLP) method. The problems of insufficiency of measurements and multiplicity of solutions are described, and appropriate approaches are proposed (introduction of additional subcriteria and uniting gas pipelines into groups). Identification of gas pipelines hydraulic efficiency coefficients for gas transmission systems of various complexity has been performed using the given algorithm.
\end{abstract}

\section{Introduction}

Significant discrepancies between the real gas transmission system (GTS) and its computer model occur quite often. On the one hand, the mathematical model cannot take into account the entire real system data. On the other hand, with time there occur changes in the GTS objects technical condition. Adaptation of mathematical models to real systems is performed by introducing appropriate empirical coefficients $[1,2]$, which are nonmeasurable parameters, into mathematical models. To determine the values of nonmeasureable parameters, the identification procedure is performed based on the actual GTS modes [3-6]. As identifiable non-measureable parameters of gas pipelines there can be hydraulic efficiency coefficients [7], hydraulic resistance coefficients [8], and roughness coefficients [9].

In the paper [10] the approach to estimation of adequacy and quality of calculated GTS modes is considered. In papers [7, 8] questions of the GTS non-measureable parameters identification are discussed. In studies [7, 8] the statistical estimation of GTS nonmeasureable parameters is performed using the maximum likelihood method by series of measurements (gas flows through GTS sections, gas pressure and temperature values at GTS nodes) for stationary and non-stationary GTS modes. One of the peculiarities of GTS non-measureable parameters identification is the multiplicity of solutions. In studies [1113 ] the term «identifiability» of parameters and quantitative assessment of the identification is given.

${ }^{*}$ Corresponding author: A.Belevitin@econom.gazprom.ru 


\section{Problem Statement}

In this paper we consider the problem of identification of GTS non-measureable parameters like gas pipelines hydraulic efficiency coefficients $\left(\mathbf{E}=\left\{E_{i}\right\}, i=1 . . K\right)$ for GTS of arbitrary configuration (including looped one, containing an arbitrary number of compressor shops) operating in the stationary non-isothermal mode.

The modeling of the gas pipeline hydraulic regime is performed according to the standard used in PJSC Gazprom [14]:

$$
p_{1, i}^{2}-p_{2, i}^{2}=\frac{\lambda_{f r, i} \cdot \Delta \cdot T_{a v, i} \cdot z_{a v, i} \cdot L_{i}}{\left(3,32 \cdot 10^{-6} \cdot d_{i}^{2,5} \cdot E_{i}\right)^{2}} \cdot\left|q_{i}\right| \cdot q_{i},
$$

where $p_{1, j}, p_{2, j}$ are pressure values at the beginning and at the end of the gas pipeline, respectively, $\mathrm{MPa} ; q_{j}$ is the gas flow through the gas pipeline, $10^{6} \mathrm{~m}^{3} /$ day; $T_{a v, j}$, is the average gas pipeline temperature value, $K ; \Delta, z_{a v, j}$ are the air relative density of gas and average gas compressibility factor, respectively; $L_{j}$ is the gas pipeline length, $k m ; d_{j}$ is the gas pipeline inside diameter, $m m ; \lambda_{f r}, E_{j}$ are the pipeline hydraulic friction resistance coefficient and hydraulic efficiency coefficient, respectively.

The initial data for the identification problem are pressure measurements at GTS nodes, gas flow rate measurements at GTS inputs and outputs (are considered fixed), gas flow measurements through GTS sections. Obligatory is the availability of gas flow rate measurements at all GTS inputs/outputs and at least one pressure measurement at GTS node. In case of the gas unbalance at GTS inputs/outputs, the balancing is performed according to the input/output, which has the maximum value of gas flow rate measurement. As additional initial data, there can be used measurements per number of revolutions of centrifugal supercharges and gas consumption of gas-compressor units for plant demand if available.

\section{Solution Procedure}

\subsection{Mathematical Model}

The GTS can be presented as a directed graph containing $M$ nodes and $N$ arcs. GTS graph nodes are points connecting arcs, or boundary conditions. GTS graph arcs are gas pipelines, compressor shops, as well as valves and connectors, which in this case are considered to be objects with zero hydraulic resistance. Suppose that $M_{l}$ nodes of $M$ contain pressure measurements and $N_{l}$ arcs of $N$ contain flow measurements.

The mathematical model for identifying gas pipelines hydraulic efficiency coefficients (GTS non-measurable parameters) includes equations describing gas movement through gas pipelines and operation of gas-compressor units, as well as Kirchhoff's first law and boundary conditions. The modelling of gas-compressor units charging mode is performed using a set of polynomials [1]. Gas consumption of a gas-compressor unit for plant demand is performed according to [15] based on individual consumption norms of the used drive.

The key unknown variables of the model are the vector of gas pipelines hydraulic efficiency coefficients E. The problem optimization criterion is the sum of squares of difference between the calculated pressure values at GTS nodes (or calculated gas flows through GTS sections) and corresponding pressure measurements (or gas flow measurements): 


$$
F_{1}(\mathbf{E})=w_{1} \cdot \sum_{i=1}^{M_{1}}\left(p_{\text {calc }, i}-p_{\text {meas }, i}\right)^{2}+w_{2} \cdot \sum_{j=1}^{N_{1}}\left(q_{\text {calc }, j}-q_{\text {meas }, j}\right)^{2} \rightarrow \min
$$

where $p_{c a l c, i}, p_{\text {meas }, i}$ are the calculated pressure value and pressure measurement at GTS node $i$, respectively; $q_{\text {calc }, j}, q_{\text {meas }, j}$ are the calculated flow value and flow measurement for the GTS arc $j$, respectively; $w_{1}, w_{2}$ are subcriteria weights.

\subsection{Model Dividing and Use of SLP method}

The considered identification problem is characterized by the high dimensionality and nonlinearity of the object function and its constraints.

Let's divide the original model into two interrelated constituents: 1) Nonlinear optimization problem; 2) Temperature calculation.

The nonlinear optimization problem comes from the original problem as a result of fixing the part of model variables: gas temperature values at nodes, gas compressibility factors and gas viscosity for pipelines. The obtained nonlinear optimization problem is solved using the method of Successive Linear Programming (SLP) [16-19]. As a result, flows are determined per GTS sections. On the basis of the calculated gas flows through GTS sections, the temperature calculation is performed. As a result, gas temperature values at GTS nodes are determined, gas compressibility factor and viscosity for gas pipelines are specified. The steps are repeated until the differences in gas flows through GTS sections, gas pressure and temperature values at GTS nodes for two adjacent iterations are below the specified accuracy values.

\subsection{Introduction of Additional Criteria}

In many cases, the existing set of measurements (gas flows through GTS sections, gas pressure and temperature values at GTS nodes) is not enough to unambiguously determine values of gas pipelines hydraulic efficiency coefficients (GTS non-measurable parameters). To reduce the number of admissible decisions, it is proposed to introduce additional subcriteria (with less weight):

1) minimum of hydraulic efficiency $E_{c a l c, i}$ deviations from $E_{i}^{*}$ values;

$-E_{i}^{*}=0.95-$ default value for hydraulic efficiency factor per [14];

- values set as initial data.

2) minimum of total commodity work [20].

Then the criterion for the problem will take the form:

$$
\begin{aligned}
& F_{2}(\mathbf{E})=w_{1} \cdot \sum_{i=1}^{M_{1}}\left(p_{\text {calc }, i}-p_{\text {meas }, i}\right)^{2}+w_{2} \cdot \sum_{j=1}^{N_{1}}\left(q_{\text {calc }, j}-q_{\text {meas }, j}\right)^{2} \\
& +w_{3} \cdot \sum_{j=1}^{K}\left(E_{\text {calc }, j}-E_{j}^{*}\right)^{2}+w_{4} \cdot \sum_{j=1}^{N}\left|q_{\text {calc }, j} \cdot L_{j}\right| \rightarrow \min
\end{aligned}
$$

where $K$ is the number of problem identifiable hydraulic efficiency coefficients; $E_{c a l c, j}$ is the calculated value of the gas pipeline $j$ hydraulic efficiency coefficient; $L_{j}$ is the length of the gas pipeline $j ; w_{3}, w_{4}$ are subcriteria weights.

\subsection{Gas Pipelines Grouping}


The program algorithm realization applies the procedure of uniting serial gas pipes with the same parameters (diameter, wall thickness, etc.), the length of the new gas pipeline being equal to the sum of lengths of the combined pipelines.

In addition, the decrease of the model unknown variables number can be performed at the cost of gas pipelines grouping - i.e. uniting various gas pipelines into groups with the single value of hydraulic efficiency coefficient. Let's consider the GTS section between two adjacent compressor stations and consider the following options:

1) compressor shops operate in the common hydraulic mode and there are no large associate gas inflows/outflows. As a result, pressure measurements at beginnings and ends of GTS sections are the same for all lines of the GTS. In this case, it is recommended to assume the values of hydraulic efficiency coefficients to be the same for all gas pipelines of that section;

2) compressor shops operate in the independent hydraulic mode (interplant valves are closed). Assume also that intermediate valves between lines are closed. Thus, each line of the GTS has an individual pressure measurement at the beginning and at the end of the section in question. In such case, it is advisable to consider gas pipelines hydraulic efficiency coefficients to be different for each line of the GTS section in question.

3 ) assume that in the previous case 2) there were open intermediate valves. In such case, it is recommended to consider gas pipelines enclosed between the nodes with pressure measurements and having a common intermediate node (open valve between the lines) to be a group of gas pipelines with the same value of hydraulic efficiency coefficient;

4) grouping of gas pipelines can be performed based on their operation life data or technical condition.

\section{Results}

Using the described approaches, calculations were performed for sections of the Unified Gas Supply System (UGSS) of Russia of varying complexity. As an example let's consider a six-line section of the UGSS of Russia called «CS Ordinskaya - CS Pochinkovskaya» (Fig. 1). That GTS contains 324 gas pipelines and 7 compressor stations (or 42 compressor shops). For this GTS, the hydraulic efficiency coefficients identification was performed.

The following actual data were used as actual measurements:

- pressure values and flow rates at all inputs and outputs of the system;

- input and output pressure values for compressor shops.

Actual revolution rates and gas consumption of gas-compressor unit were not available.

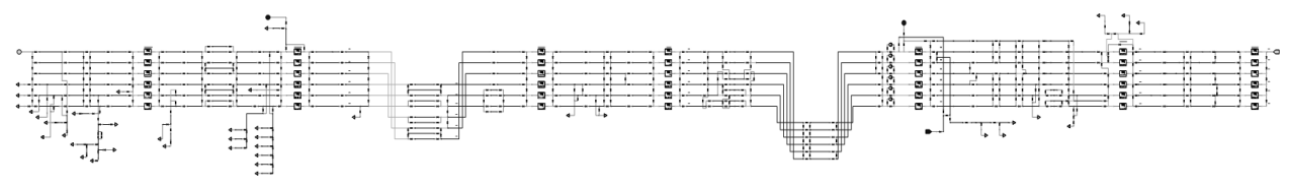

Fig. 1. Calculated gas transmission system

The criterion (3) was used with the following weights: $w_{1}=1.0, w_{2}=0$ (there were no flow measurements for arcs), $w_{3}=0.01, w_{4}=0.000001$. The value $E_{j}^{*}=0.95$ (3) was used.

Gas pipelines were united into groups with the single value of hydraulic efficiency coefficient based on the given recommendations (subsection 3.4), but without using information about their operation life and technical condition. The examples of uniting gas pipelines into groups for two different sections of the GTS between adjacent compressor stations are shown on Fig. 2 (a) and (b).

Table 1 shows the number of gas pipelines and the number of model unknown variables (gas pipelines hydraulic efficiency coefficients) for the following cases: the initial variant (without gas pipelines interconnection and combining into groups), after interconnection of 
successive gas pipelines with the same parameters and after combining gas pipelines into groups with the single value of hydraulic efficiency coefficient. After interconnection of successive gas pipelines, the number of gas pipelines and the number of model unknown variables significantly decreased and remained equal. Combining gas pipelines into groups has led to the fact that the number of gas pipelines remained the same, but the number of the model unknown variables significantly decreased.

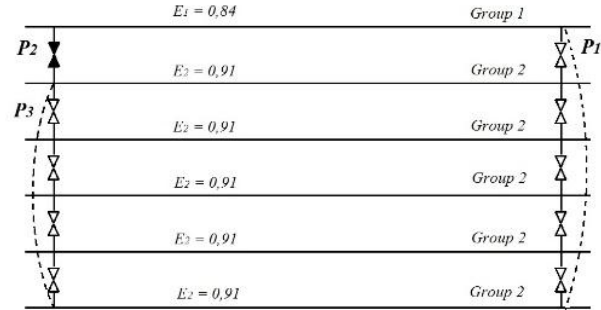

(a)

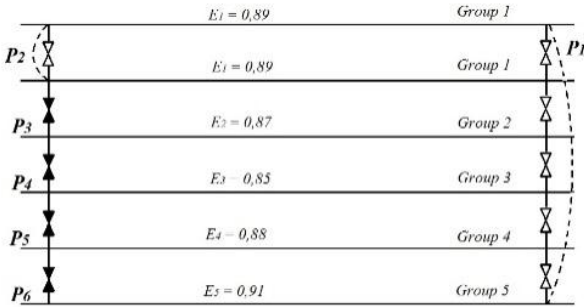

(b)

Fig. 2. Examples of uniting gas pipelines into groups for two different (a) and (b) sections of the GTS between adjacent compressor stations

Table 1. Number of gas pipelines and number of model unknown variables

\begin{tabular}{|c|c|c|c|}
\hline Parameter & Initially & $\begin{array}{c}\text { After merging of } \\
\text { successive gas } \\
\text { pipelines }\end{array}$ & $\begin{array}{c}\text { After combining gas } \\
\text { pipelines in groups }\end{array}$ \\
\hline $\begin{array}{c}\text { Number of gas } \\
\text { pipelines }\end{array}$ & 324 & 140 & 140 \\
\hline $\begin{array}{c}\text { Number of model } \\
\text { unknown variables }\end{array}$ & 324 & 140 & 63 \\
\hline
\end{tabular}

Fig. 3 shows the values of the calculated gas pipelines hydraulic efficiency coefficients for two cases: without and with interconnection and uniting of gas pipelines into groups.

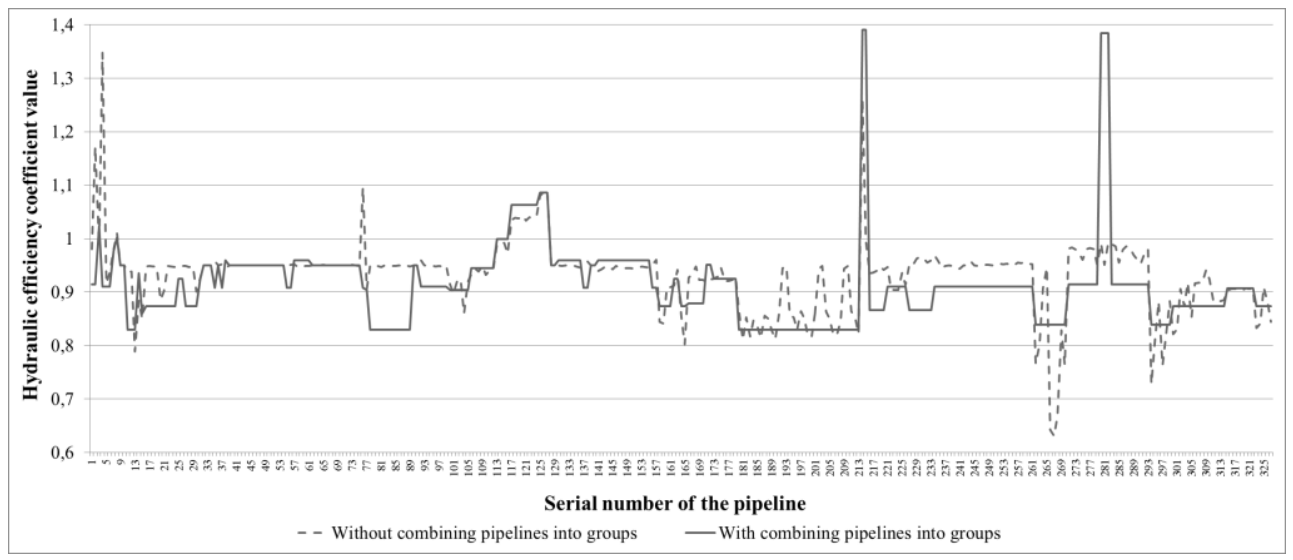

Fig. 3. The results of the gas pipelines hydraulic efficiency coefficients identification 


\section{Conclusions}

This study presents the problem statement and solution procedure for identifying GTS nonmeasureable parameters - gas pipelines hydraulic efficiency coefficients. There have been proposed approaches to reducing the number of problem solutions at the cost of introducing additional criteria and uniting gas pipelines into groups. The problem of the gas pipelines hydraulic efficiency coefficients identification is considered to be an optimization problem. To solve it, the method of successive linear programming (SLP) is used. As an example, the calculation of the section of the UGSS of Russia was examined. There were compared the results both without and with interconnection and uniting of gas pipelines into groups.

\section{References}

1. S.A. Sardanashvili, Calculation methods and algorithms (pipeline gas transportation) (Oil and Gas, Moscow, 2005)

2. M.G. Sukharev, A.M. Karasevich, Technological calculation and reliability control of gas and oil pipelines (Oil and Gas, Moscow, 2000)

3. M.A. Kanibolotsky, Identification of parameters of gas production and transportation systems (Ph.D. thesis, Novosibirsk, 1975)

4. V.G. Sidler, Development and application of methods for identification of hydraulic network parameters (Ph.D. thesis, Tomsk, 1977)

5. N.N. Novitsky, Evaluation of hydraulic circuit parameters (Science, Novosibirsk, 1988)

6. M.G. Sukharev, R.V. Samoilov, Analysis and control of stationary and non-stationary modes of gas transportation (GRSU of Oil and Gas, Moscow, 2016)

7. M.G. Sukharev, K.O. Kosova, Autom. Remote Control. 78, 882 (2017)

8. M.G. Sukharev, K.O. Kosova, Proceedings of Gubkin Russian State University of Oil and Gas, 3(276), 319 (2014)

9. https://www.researchgate.net/publication/322055623_PIPELINE_OPTIMIZATIONA_SURFACE_ROUGHNESS_APPROACH (access date: December, 2017)

10. S.A. Sardanashvili, V.V. Samsonova, Gas Industry, 9, 84 (2013)

11. N.N. Novitsky, Methods of analysis and optimal synthesis of pipeline systems, 142 (ESI SB RAS, Irkutsk, 1991)

12. N.N. Novitsky, System research in power engineering: a retrospective of scientific trends, 279 (Science, Novosibirsk, 2010)

13. N.N. Novitsky, O.A. Grebneva, E3S Web of Conferences, 39, 03004 (2018)

14. Company Standard STO Gazprom 2-3.5-051-2006 (PJSC Gazprom, Moscow, 2016)

15. Company Standard STO Gazprom 3.3-2-024-2011 (PJSC Gazprom, Moscow, 2011)

16. R. Griffith, R. Stewart, Management Science, 7, 379 (1961)

17. D. Himmelblau, Applied Nonlinear Programming (Mir publishers, Moscow, 1975)

18. J. Zang, A successive linear programming method and its convergence on nonlinear problems (Austin, Texas, 1983)

19. M.S. Bazaraa, H.D. Sherali, C.M. Shetty, Nonlinear Programming: Theory and Algorithms (Wiley, N.-Y, 2006)

20. Company Standard STO Gazprom 2-3.5-113-2007 (PJSC Gazprom, Moscow, 2007) 